\title{
Current-conserving and gauge-invariant quantum ac transport theory in the presence of phonon
}

\author{
Yunjin Yu, ${ }^{1}$ Hongxin Zhan, ${ }^{1}$ Yadong Wei, ${ }^{1, *}$ and Jian Wang ${ }^{2, *}$ \\ ${ }^{1}$ College of Physics and Institute of Computational Condensed Matter Physics, Shenzhen University, \\ Shenzhen 518060, People's Republic of China \\ ${ }^{2}$ Department of Physics and the Center of Theoretical and Computational Physics, The University of Hong Kong, \\ Pokfulam Road, Hong Kong, People's Republic of China
}

(Received 16 April 2014; revised manuscript received 17 June 2014; published 8 August 2014)

\begin{abstract}
Using the nonequilibrium Green's function (NEGF) approach, we develop a microscopic ac transport theory in the presence of electron-phonon interaction. Taking into account the self-consistent Coulomb interaction, the displacement current is included. This ensures that our theory satisfies the current-conserving and gauge-invariant conditions. Importantly, the inclusion of self-consistent Coulomb interaction naturally connects the NEGF formalism to the density functional theory (DFT). This allows us to calculate the self-consistent Hamiltonian using DFT within the NEGF framework, which paves the way for first principles ac transport calculation of nanoelectronic devices in the presence of electron-phonon interaction. It is known that the inelastic electron tunneling spectroscopy (IETS) is a powerful tool in studying the inelastic de quantum transport in molecular devices. The basic idea of IETS is to obtain the information of vibrational spectrum of molecular devices by measuring the second derivative of the dc current with respect to the bias voltage. In the ac transport, we find that the phonon spectrum and electron-phonon coupling strength can be obtained from the second derivative of the admittance with respect to the frequency which is the working principle of the inelastic electron admittance spectroscopy (IEAS). Hence we propose to use IEAS to probe the effect of the phonon in ac transport. As an example, dynamic conductance of a quantum dot is discussed in detail and the concept of IEAS is demonstrated.
\end{abstract}

DOI: 10.1103/PhysRevB.90.075407

PACS number(s): 72.10.Di, 72.25.-b, 85.65.+h

\section{INTRODUCTION}

Quantum transport in nanostructures has been intensively investigated because of promising potential applications in nanoelectronics [1-3]. Various transport properties including $I-V$ characteristic, conductivity, current noise, quantum capacitance, etc. have been studied both experimentally [4-9] and theoretically [10-16]. Although most theoretical and experimental studies have been concentrated on the dc transport regime, time-dependent transport problems have attracted more and more attention including steady state ac transport [17-32] and the transient problems driven by the step function-like bias [33,34]. Ac quantum transport properties have also been investigated in the super-conducting hybrid system [35-37]. To examine the phase breaking effect, ac quantum transport with electron-phonon interaction has been studied by several groups $[21,38,39]$. For electron transport, current-conserving and gauge-invariant conditions are two fundamental requirements $[10,11]$. In order to satisfy the two conditions in ac transport, one should include self-consistent Coulomb interaction explicitly into the Hamiltonian and calculate both conduction current and displacement current due to the charge pileup in the scattering region $[10,11,23,24]$. Moreover, the inclusion of Coulomb interaction is also the key for the first principles transport calculation for nanoelectronics which bridges the nonequilibrium Green's function in quantum transport theory (NEGF) and the density function theory (DFT) characterizing the chemical ingredient of the molecular devices [40].

One of the most important issues in nanoelectronics theory and modeling is the role played by interaction between

*Corresponding authors: ywei@szu.edu.cn; jianwang@hku.hk electrons and the nuclear vibrations (phonons). This is because the electron-phonon interaction gives rise to inelastic current and modifies the elastic current that affects the characteristics of nanoelectronic devices in an essential way. In addition, the inelastic current due to electron-phonon interaction can be used to measure vibrational spectra of single molecule as has been demonstrated experimentally using inelastic tunneling spectroscopy. Clearly, predicting quantum transport properties of nanoelectronic devices in the presence of electron-phonon interaction must use the first principle method including all the atomic details of nanoelectronic devices.

Indeed, various effects on the transport in the molecular devices have been studied such as inelastic electron scattering, atomic position rearrangements [3], energy dissipation [41-43] and local heat in device [44], phonon-mediated negative differential resistance [45] and phonon assisted tunneling $[9,46]$, etc. In the dc situation, the inelastic transport properties of molecular device are studied intensively [33,47-54]. The inelastic tunneling spectroscopy (IETS), which is the second derivative of the current over bias voltage, is usually used as a powerful tool to identify the molecular vibrational modes and the electron-phonon coupling strength $[55,56]$. These first principle investigations show that under nonequilibrium condition vibrational spectra and electron-phonon interaction can be quite different from the equilibrium ones. For instance, it was found that the dc bias voltage can drastically affect the electron-phonon coupling strength while the phonon frequencies change only a few percent [56]. Since most of the nanoelectronic devices are operated with ac signals and finite temperatures, there is a clear need to understand the role played by electron-phonon interaction in the ac regime. Up to now, most of the theoretical studies of electron-phonon interaction focus on dc properties [57]. There is yet a first principle method for calculating vibrational frequencies, electron-phonon 
couplings, and inelastic transport properties under ac condition for nanoelectronic devices. It is the aim of this paper to provide such a theoretical framework which is suitable for the first principles calculation in nanoelectronics. In this paper, we developed a microscopic theory for ac transport with electronphonon coupling using the nonequilibrium Green's function theory. Our formalism emphasizes the current conservation and gauge invariance. The self-consistent Coulomb interaction in the presence of phonon is included explicitly in the Hamiltonian making it possible to combine with DFT for the first principles transport calculation. Similar to the role IETS played in the dc transport, we proposed a new tool named as the ac inelastic electron admittance spectroscopy (IEAS) to characterize the electron-phonon interaction including phonon spectrum and electron-phonon coupling strength from the dynamic point of view with frequency as an extra handle.

The rest of the paper is organized as follows. In Sec. II, the formulas of current and conductance are derived for quantum systems with ac bias and electron-phonon interaction. Using the nonequilibrium Green's function, the dynamic conductance is obtained by expanding ac current and admittance to the first order with respect to the amplitude of ac bias. In addition, current conservation and gauge invariance are proved to be satisfied when the Coulomb interaction is considered. Furthermore, the idea of IEAS is introduced and analyzed. In Sec. III, as an example, the inelastic ac transport of a quantum dot is numerically studied in detail. A summary is given in Sec. IV.

\section{THEORETICAL FORMALISM}

We consider a scattering region coupled by two or multiple leads. The system can be described by the following Hamiltonian:

$$
H=H_{\text {lead }}+H_{\text {scat }}+H_{T} .
$$

$H_{\text {lead }}$ is the Hamiltonian of the leads,

$$
H_{\text {lead }}=\sum_{k \alpha} \epsilon_{k \alpha} \hat{C}_{k \alpha}^{\dagger} \hat{C}_{k \alpha}
$$

$\hat{C}_{k \alpha}^{\dagger}\left(\hat{C}_{k \alpha}\right)$ is the creation (annihilation) operator of the $k$ state in the lead $\alpha$ ( $\alpha=L, R$ for a two-probe system). $\epsilon_{k \alpha}=\epsilon_{k \alpha}^{(0)}+$ $q v_{\alpha} \cos \omega t$ with $\epsilon_{k \alpha}^{(0)}$ the energy level in lead $\alpha$ and $v_{\alpha}$ is the ac bias amplitude on the lead $\alpha$. As usual, the electron-phonon interaction and Coulomb interaction in the lead are neglected.

$H_{\text {scat }}$ is the Hamiltonian of the scattering region which includes three parts as follows:

$$
H_{\text {scat }}=H_{e}+H_{p}+H_{e p} .
$$

The Hamiltonian of electrons $H_{e}$ in the scattering region can be expressed as

$$
H_{e}=\sum_{n}\left(\epsilon_{n}+q U_{n}\right) \hat{d}_{n}^{\dagger} \hat{d}_{n},
$$

where $\hat{d}_{n}^{\dagger}$ and $\hat{d}_{n}$ are the creation and annihilation operators in the scattering region and satisfy the fermion anticommutation relation $\left\{\hat{d}_{\alpha}, \hat{d}_{\beta}^{\dagger}\right\}=\delta_{\alpha \beta} . U_{n}=\sum_{m} V_{n m}\left\langle\hat{d}_{m}^{\dagger} \hat{d}_{m}\right\rangle$ is the self-consistent internal Coulomb potential inside the scattering region with $V_{n m}$ the matrix element of the Coulomb potential
[58]. If we wish to implement this formalism into the first principles calculation, we will use the following Hamiltonian that includes the potentials $V_{\mathrm{ex}}$ and $V_{\text {cor }}$ due to the the exchange and correlation interactions, respectively:

$$
H_{e}=\sum_{n}\left(\epsilon_{n}+q U_{n}+q V_{\mathrm{ex}}+q V_{\mathrm{cor}}\right) \hat{d}_{n}^{\dagger} \hat{d}_{n} .
$$

In Eq. (3), $H_{p}$ is the phonon Hamiltonian and can be written as

$$
H_{p}=\sum_{\nu} \hbar \omega_{\nu}\left(\hat{b}_{\nu}^{\dagger} \hat{b}_{\nu}+\frac{1}{2}\right)
$$

where $\omega_{\nu}$ is the phonon frequency. The phonon creation and annihilation operators, $\hat{b}_{v}^{\dagger}$ and $\hat{b}_{v}$, satisfy the boson commutation relation $\left[\hat{b}_{\alpha}, \hat{b}_{\beta}^{\dagger}\right]=\delta_{\alpha \beta}$. The Hamiltonian of the electron-phonon interaction is given by

$$
H_{e p}=\sum_{v n n^{\prime}} g_{n n^{\prime}}^{v}\left(\hat{b}_{v}^{\dagger}+\hat{b}_{v}\right) \hat{d}_{n}^{\dagger} \hat{d}_{n^{\prime}},
$$

where $g_{n n^{\prime}}^{v}$ describes the electron-phonon coupling strength.

The third term $H_{T}$ in the total Hamiltonian Eq. (1) describes the coupling between the scattering region and the leads. With the coupling constant $t_{k \alpha n}$, it can be expressed as

$$
H_{T}=\sum_{k \alpha n}\left[t_{k \alpha n} \hat{C}_{k \alpha}^{\dagger} \hat{d}_{n}+t_{k \alpha n}^{*} \hat{d}_{n}^{\dagger} \hat{C}_{k \alpha}\right]
$$

Using the Heisenberg equation of motion, one obtains the current in the form of Green's function

$$
I_{\alpha}(t)=-\sum_{k n}\left[t_{k \alpha n} G_{n, k \alpha}^{<}(t, t)\right]+\text { H.c. }
$$

After the analytic continuation, we obtain [14]

$$
\begin{aligned}
I_{\alpha}(t)= & -q \int d t_{1} \operatorname{Tr}\left[G^{r}\left(t, t_{1}\right) \Sigma_{e \alpha}^{<}\left(t_{1}, t\right)+G^{<}\left(t, t_{1}\right) \Sigma_{e \alpha}^{a}\left(t_{1}, t\right)\right. \\
& \left.-\Sigma_{e \alpha}^{<}\left(t, t_{1}\right) G^{a}\left(t_{1}, t\right)-\Sigma_{e \alpha}^{r}\left(t, t_{1}\right) G^{<}\left(t_{1}, t\right)\right],
\end{aligned}
$$

where

$$
\Sigma_{e \alpha m n}^{\gamma}\left(t, t^{\prime}\right)=\sum_{k} t_{k \alpha m}^{*} g_{k \alpha}^{\gamma}\left(t, t^{\prime}\right) t_{k \alpha n},
$$

with $\gamma=<, r, a$. Here $\Sigma_{e \alpha}^{\gamma}$ is the self-energy due to the electron coupling between the scattering region and the lead $\alpha$. Electron-phonon coupling between the scattering region and the leads is set to zero since we assume that the phonon exists only in the scattering region but not in the lead regions. In Eq. (11), the Green's functions of isolated leads are

$$
g_{k \alpha}^{r, a}\left(t, t^{\prime}\right)=\mp i \theta\left( \pm t \mp t^{\prime}\right) \exp \left[-i \int_{t^{\prime}}^{t} d t_{1} \epsilon_{k \alpha}\left(t_{1}\right)\right]
$$

and

$$
g_{k \alpha}^{<}\left(t, t^{\prime}\right)=i f\left(\epsilon_{k \alpha}^{(0)}\right) \exp \left[-i \int_{t^{\prime}}^{t} d t_{1} \epsilon_{k \alpha}\left(t_{1}\right)\right] .
$$

The effect of phonon is included in the Green's function $G^{\gamma}$ in Eq. (10) as self-energy $\Sigma_{e p}^{\gamma}$ which will be discussed in detail in Sec. II B. 


\section{A. Nonequilibrium Green's function at small bias}

We are interested in the linear response regime where the bias voltage is small. To calculate the ac current and dynamic conductance in this regime, one can expand the Green's function $G^{\gamma}$, electronic self-energy $\Sigma_{e}^{\gamma}$, and the self-energy of electron-phonon coupling $\Sigma_{e p}^{\gamma}$ to the first order of the external bias $v_{\alpha}$ as follows:

$$
\begin{gathered}
G^{\gamma}\left(t, t_{1}\right)=G_{0}^{\gamma}\left(t, t_{1}\right)+g^{\gamma}\left(t, t_{1}\right), \\
\Sigma_{e \alpha}^{\gamma}\left(t, t_{1}\right)=\Sigma_{0 e \alpha}^{\gamma}\left(t, t_{1}\right)+\sigma_{e \alpha}^{\gamma}\left(t, t_{1}\right),
\end{gathered}
$$

and

$$
\Sigma_{e p}^{\gamma}\left(t, t_{1}\right)=\Sigma_{0 e p}^{\gamma}\left(t, t_{1}\right)+\sigma_{e p}^{\gamma}\left(t, t_{1}\right)
$$

where $G_{0}^{\gamma}, \Sigma_{0 e \alpha}^{\gamma}$, and $\Sigma_{0 e p}^{\gamma}$ are equilibrium Green's functions and equilibrium self-energies and $g^{\gamma}, \sigma_{e \alpha}^{\gamma}$, and $\sigma_{e p}^{\gamma}$ are the first order corrections due to the bias $v_{\alpha}$. It is straightforward to find that the current in the linear regime is written as

$$
\begin{aligned}
I_{\alpha}(t)= & -q \int d t_{1} \operatorname{Tr}\left[G_{0}^{r}\left(t, t_{1}\right) \sigma_{e \alpha}^{<}\left(t_{1}, t\right)+g^{r}\left(t, t_{1}\right) \Sigma_{0 e \alpha}^{<}\left(t_{1}, t\right)\right. \\
& +G_{0}^{<}\left(t, t_{1}\right) \sigma_{e \alpha}^{a}\left(t_{1}, t\right)+g^{<}\left(t, t_{1}\right) \Sigma_{0 e \alpha}^{a}\left(t_{1}, t\right) \\
& -\Sigma_{0 e \alpha}^{<}\left(t, t_{1}\right) g^{a}\left(t_{1}, t\right)-\sigma_{e \alpha}^{<}\left(t, t_{1}\right) G_{0}^{a}\left(t_{1}, t\right) \\
& \left.-\Sigma_{0 e \alpha}^{r}\left(t, t_{1}\right) g^{<}\left(t_{1}, t\right)-\sigma_{e \alpha}^{r}\left(t, t_{1}\right) G_{0}^{<}\left(t_{1}, t\right)\right] .
\end{aligned}
$$

After taking double-time Fourier transform, the expression of current in energy representation is obtained as

$$
\begin{aligned}
I_{\alpha}(\Omega)= & -q \int \frac{d E}{2 \pi} \operatorname{Tr}\left[G_{0}^{r}\left(E_{+}\right) \sigma_{e \alpha}^{<}\left(E_{+}, E\right)+g^{r}\left(E_{+}, E\right)\right. \\
& \times \Sigma_{0 e \alpha}^{<}(E)+G_{0}^{<}\left(E_{+}\right) \sigma_{e \alpha}^{a}\left(E_{+}, E\right)+g^{<}\left(E_{+}, E\right) \Sigma_{0 e \alpha}^{a}(E) \\
& -\Sigma_{0 e \alpha}^{<}\left(E_{+}\right) g^{a}\left(E_{+}, E\right)-\sigma_{e \alpha}^{<}\left(E_{+}, E\right) G_{0}^{a}(E) \\
& \left.-\Sigma_{0 \alpha}^{r}\left(E_{+}\right) g^{<}\left(E_{+}, E\right)-\sigma_{e \alpha}^{r}\left(E_{+}, E\right) G_{0}^{<}(E)\right]
\end{aligned}
$$

where $E_{+}=E+\Omega$. (We will set $\hbar=1$ in the rest of the paper.) To obtain Eq. (18), we have used the fact that Fourier transform $G_{0}^{\gamma}\left(E, E^{\prime}\right)=2 \pi \delta\left(E-E^{\prime}\right) G_{0}^{\gamma}(E)$ and similar relation for the equilibrium self-energy. This is because the equilibrium Green's function $G_{0}^{\gamma}\left(t_{1}, t_{2}\right)$ and self-energy $\Sigma_{0 e}^{\gamma}\left(t_{1}, t_{2}\right)$ depend only on the time difference $t_{1}-t_{2}$.

In the energy representation, the expression of the retarded Green's function is [59]

$$
G^{r}=\frac{1}{E-H_{0}-U-\Sigma_{e}^{r}-\Sigma_{e p}^{r}},
$$

where $H_{0}=\sum_{n} \epsilon_{n} \hat{d}_{n}^{\dagger} \hat{d}_{n}$ is the Hamiltonian of isolated scattering region, $U$ is the self-consistent internal Coulomb potential, and $\Sigma_{e p}^{r}$ is the phonon self-energy to be discussed in detail in the next subsection. Expanding $U(t)$ in terms of the amplitude of the external bias $v_{\alpha}(0)=v_{\alpha}$, we have

$$
\begin{aligned}
U(t) & =U_{\text {eq }}+U_{1}(t)+U_{2}(t)+\cdots \\
& =U_{\text {eq }}+\sum_{\alpha} u_{\alpha}(t) v_{\alpha}+\frac{1}{2} \sum_{\alpha \beta} u_{\alpha \beta}(t) v_{\alpha} v_{\beta}+\cdots,
\end{aligned}
$$

where $U_{\text {eq }}$ is the equilibrium Coulomb potential and $u_{\alpha}(t)$, $u_{\alpha \beta}(t)$ are the so-called characteristic potentials [11,25]. Here $u_{\alpha}(t)$ corresponds to the first order response of the Coulomb interaction due to ac bias and $u_{\alpha \beta}(t)$ describes the second order correction, etc. According to the gauge-invariant condition the current should remain unchanged when all the external bias voltages are shifted to an equal amount at the same time; we have $\sum_{\alpha} u_{\alpha}(t)=\cos \omega t$ and $\sum_{\alpha} u_{\alpha \beta}(t)=\sum_{\beta} u_{\alpha \beta}(t)=0$ in the presence of phonon [11,25].

In the linear response regime, we treat $U_{1}, \sigma_{e}^{r}$, and $\sigma_{e p}^{r}$ as the perturbation to the equilibrium quantity $U_{\text {eq }}, \Sigma_{0 e}^{r}$, and $\Sigma_{0 e p}^{r}$, respectively. We have from the Dyson equation

$$
G^{r}=G_{0}^{r}+G_{0}^{r}\left(U_{1}+\sigma_{e}^{r}+\sigma_{e p}^{r}\right) G_{0}^{r},
$$

where the equilibrium Green's function $G_{0}^{r}$ is

$$
G_{0}^{r}=\frac{1}{E-H_{0}-U_{\mathrm{eq}}-\Sigma_{0 e}^{r}-\Sigma_{0 e p}^{r}} .
$$

So from Eqs. (14) and (21), the first order correction for the retarded Green's function is

$$
g^{r / a}=G_{0}^{r / a}\left(U_{1}+\sigma_{e}^{r / a}+\sigma_{e p}^{r / a}\right) G_{0}^{r / a} .
$$

Using the Keldysh equation $G^{<}=G^{r} \Sigma^{<} G^{a}$, and collecting the first order terms of the external bias, one finds

$$
g^{<}=G_{0}^{r} \Sigma_{0}^{<} g^{a}+G_{0}^{r} \sigma^{<} G_{0}^{a}+g^{r} \Sigma_{0}^{<} G_{0}^{a},
$$

where

$$
\Sigma_{0}^{<}=\Sigma_{0 e}^{<}+\Sigma_{0 e p}^{<}
$$

and

$$
\sigma^{<}=\sigma_{e}^{<}+\sigma_{e p}^{<} .
$$

Note that Eqs. (23) and (24) are in time space. Taking double-time Fourier transform of these two equations and using the abbreviation $\bar{G}_{0}^{\gamma}=G_{0}^{\gamma}\left(E_{+}\right)$and $\bar{\Sigma}_{0}^{\gamma}=\Sigma_{0}^{\gamma}\left(E_{+}\right)$with $E_{+}=E+\Omega$, we have

$$
\begin{aligned}
g^{r / a}\left(E_{+}, E\right)= & \bar{G}_{0}^{r / a}\left[U_{1}(\Omega)+\sigma_{e}^{r / a}\left(E_{+}, E\right)\right. \\
& \left.+\sigma_{e p}^{r / a}\left(E_{+}, E\right)\right] G_{0}^{r / a}
\end{aligned}
$$

and

$$
\begin{aligned}
g^{<}\left(E_{+}, E\right)= & \bar{G}_{0}^{r}\left(\bar{\Sigma}_{0 e}^{<}+\bar{\Sigma}_{0 e p}^{<}\right) g^{a}\left(E_{+}, E\right)+\bar{G}_{0}^{r}\left[\sigma_{e}^{<}\left(E_{+}, E\right)\right. \\
& \left.+\sigma_{e p}^{<}\left(E_{+}, E\right)\right] G_{0}^{a}+g^{r}\left(E_{+}, E\right)\left(\Sigma_{0 e}^{<}+\Sigma_{0 e p}^{<}\right) G_{0}^{a} .
\end{aligned}
$$

The Fourier transform of the first order correction of the nonequilibrium self-energy is [21]

$$
\sigma_{e \alpha}^{\gamma}=\frac{q v_{\alpha}(\Omega)}{\Omega}\left[\Sigma_{0 e \alpha}^{\gamma}-\bar{\Sigma}_{0 e \alpha}^{\gamma}\right]
$$

where $v_{\alpha}(\Omega)=\pi v_{\alpha}[\delta(\Omega+\omega)+\delta(\Omega-\omega)]$. Here $\omega$ is the driving frequency and $\Omega$ is the response frequency. In the equations above, $U_{1}(\Omega)$ is the Fourier transform of $U_{1}(t)$ with

$$
U_{1}(\Omega)=\sum_{\alpha} u_{\alpha}(\Omega) v_{\alpha}(\Omega)
$$

From $\sum_{\alpha} u_{\alpha}(t)=\cos \omega t$, we obtain

$$
\sum_{\alpha} u_{\alpha}(\Omega)=1 .
$$

Note that here $u_{\alpha}(\Omega)$ is defined by Eq. (29) but not the direct Fourier transform of $u_{\alpha}(t)$. The expressions of $\Sigma_{0 e p}^{\gamma}$ and $\sigma_{e p}^{\gamma}$ will be derived in the next subsection. 


\section{B. Self-energy due to electron-phonon coupling}

It can be extremely computationally demanding to include the effect of electron-phonon coupling in the quantum transport. This is because the electron distribution function and electron transport are affected by the presence of phonons which in turn influence the phonon distribution function and the equilibrium positions of the atoms. So to solve this inelastic transport thoroughly, one should carry out the self-consistent calculation to get the electron density, the Coulomb interaction, as well as the electron-phonon coupling including the positions of atoms and the vibration modes. This makes the calculation extremely hard if not impossible. To reduce the complexity of the problem, only the lowest-order contributions due to electron-phonon coupling are usually considered [39,53,60-62]. At this level, if the phonon calculation is decoupled with the electronic part, we call it the Born approximation. Otherwise, it is called the self-consistent Born approximation.

In order to proceed further, we assume that $[56,63]$ the phonon is in equilibrium and its lifetime is infinite. Under this approximation, we can write the bare phonon Green's function as [64]

$$
\begin{aligned}
& D_{\nu}^{r}(\Omega)=\frac{1}{\Omega-\omega_{v}+i \epsilon}-\frac{1}{\Omega+\omega_{v}+i \epsilon}, \\
& D_{v}^{<}(\Omega)=-2 \pi i\left[n_{v} \delta\left(\Omega-\omega_{v}\right)+\left(n_{v}+1\right) \delta\left(\Omega+\omega_{\nu}\right)\right], \\
& D_{v}^{>}(\Omega)=-2 \pi i\left[\left(n_{v}+1\right) \delta\left(\Omega-\omega_{v}\right)+n_{v} \delta\left(\Omega+\omega_{\nu}\right)\right],
\end{aligned}
$$

where

$$
n_{v}=\frac{1}{e^{\omega_{v} / k_{B} T}-1}
$$

is the Bose-Einstein distribution function and $D_{v}^{a}(\Omega)=$ $\left[D_{v}^{r}(\Omega)\right]^{\dagger}$. Within the self-consistent Born approximation (SCBA), the electron self-energy due to electron-phonon coupling can be written as [64]

$$
\Sigma_{e p}\left(\tau, \tau^{\prime}\right)=i \sum_{\nu} g^{\nu} G\left(\tau, \tau^{\prime}\right) g^{\nu \dagger} D_{\nu}\left(\tau, \tau^{\prime}\right),
$$

where $D_{v}$ is the equilibrium phonon Green's function and only depends on the time difference $\tau-\tau^{\prime}$. After performing the Fourier transform, we have

$$
\Sigma_{e p}\left(E, E^{\prime}\right)=i \sum_{\nu} \int \frac{d \Omega}{2 \pi} g^{\nu} G\left(E-\Omega, E^{\prime}-\Omega\right) g^{\nu \dagger} D(\Omega),
$$

where $g^{\nu}$ is the electron-phonon coupling matrix. Its first order correction to the external bias can be written as [21]

$$
\sigma_{e p}\left(E, E^{\prime}\right)=i \sum_{v} \int \frac{d \Omega}{2 \pi} g^{v} g\left(E-\Omega, E^{\prime}-\Omega\right) g^{\nu^{\dagger}} D(\Omega) \text {. }
$$

From Eq. (34), the equilibrium self-energies due to electronphonon coupling can be solved as [63]

$$
\begin{aligned}
\Sigma_{0 e p}^{r}(E)= & \sum_{\nu} g^{\nu}\left[\left(1+n_{\nu}\right) G_{0}^{r}\left(E-\omega_{\nu}\right)+n_{\nu} G_{0}^{r}\left(E+\omega_{\nu}\right)\right. \\
& \left.+\frac{1}{2}\left[G_{0}^{<}\left(E-\omega_{\nu}\right)-G_{0}^{<}\left(E+\omega_{\nu}\right)\right]\right] g^{\nu \dagger}
\end{aligned}
$$

$$
\begin{aligned}
\Sigma_{0 e p}^{<}(E)= & \sum_{\nu} g^{\nu}\left[\left(1+n_{\nu}\right) G_{0}^{<}\left(E+\omega_{\nu}\right)\right. \\
& \left.+n_{\nu} G_{0}^{<}\left(E-\omega_{\nu}\right)\right] g^{\nu \dagger}
\end{aligned}
$$

and

$$
\begin{aligned}
\Sigma_{0 e p}^{>}(E)= & \sum_{\nu} g^{\nu}\left[\left(1+n_{\nu}\right) G_{0}^{>}\left(E-\omega_{\nu}\right)\right. \\
& \left.+n_{\nu} G_{0}^{<}\left(E+\omega_{\nu}\right)\right] g^{\nu \dagger} .
\end{aligned}
$$

At zero temperature, $n_{v}=0$, and the expressions above can be simplified as

$$
\begin{gathered}
\Sigma_{0 e p}^{r}(E)=\sum_{\nu} g^{\nu}\left[G_{0}^{r}\left(E-\omega_{\nu}\right)+\frac{1}{2} G_{0}^{<}\left(E-\omega_{\nu}\right)\right. \\
\left.\quad-\frac{1}{2} G_{0}^{<}\left(E+\omega_{\nu}\right)\right] g^{v \dagger} \\
\Sigma_{0 e p}^{<}(E)=\sum_{\nu} g^{v} G_{0}^{<}\left(E+\omega_{\nu}\right) g^{\nu \dagger}
\end{gathered}
$$

and

$$
\Sigma_{0 e p}^{>}(E)=\sum_{\nu} g^{\nu} G_{0}^{>}\left(E-\omega_{\nu}\right) g^{\nu \dagger} .
$$

Here, the Green's functions are the equilibrium Green's functions which also include the electron-phonon interaction.

At zero temperature, the first order correction of phonon self-energies can be written as

$$
\begin{aligned}
\sigma_{e p}^{r}\left(E_{+}, E\right)= & \sum_{\nu} g^{v}\left[g^{r}\left(E_{+}-\omega_{\nu}, E-\omega_{\nu}\right)\right. \\
& +\frac{1}{2} g^{<}\left(E_{+}-\omega_{\nu}, E-\omega_{\nu}\right) \\
& \left.-\frac{1}{2} g^{<}\left(E_{+}+\omega_{\nu}, E+\omega_{\nu}\right)\right] g^{v \dagger}, \\
\sigma_{e p}^{<}\left(E_{+}, E\right)= & \sum_{\nu} g^{v} g^{<}\left(E_{+}+\omega_{\nu}, E+\omega_{\nu}\right) g^{\nu \dagger},
\end{aligned}
$$

and

$$
\sigma_{e p}^{>}\left(E_{+}, E\right)=\sum_{\nu} g^{\nu} g^{>}\left(E_{+}-\omega_{\nu}, E-\omega_{\nu}\right) g^{\nu \dagger} .
$$

We note that the first order correction of phonon self-energies cannot be expressed analytically because Green's functions $g^{\gamma}$ themselves contain the phonon self-energy, so they can only be solved numerically. With the phonon self-energies defined, we are able to calculate dynamic conductance in the presence of phonon.

\section{Dynamic conductance}

The dynamic conductance (admittance) $G_{\alpha \beta}$ is defined as

$$
I_{\alpha}(\Omega)=\sum_{\beta} G_{\alpha \beta}(\Omega) v_{\beta}(\Omega) .
$$

Comparing Eq. (18) with Eq. (45) and defining $g_{\alpha}^{\gamma}(\Omega)$ and $\sigma_{\alpha}^{\gamma}(\Omega)$ according to

$$
g^{\gamma}\left(E_{+}, E\right)=\sum_{\alpha} g_{\alpha}^{\gamma}(\Omega) v_{\alpha}(\Omega)
$$


and

$$
\sigma^{\gamma}\left(E_{+}, E\right)=\sum_{\alpha} \sigma_{\alpha}^{\gamma}(\Omega) v_{\alpha}(\Omega),
$$

the dynamic conductance is found to be

$$
\begin{aligned}
G_{\alpha \beta}(\Omega)= & -q \int \frac{d E}{2 \pi} \operatorname{Tr}\left[g_{\beta}^{<}\left(\Sigma_{e \alpha}^{a}-\bar{\Sigma}_{e \alpha}^{r}\right)+g_{\beta}^{r} \Sigma_{e \alpha}^{<}-\bar{\Sigma}_{e \alpha}^{<} g_{\beta}^{a}\right. \\
& \left.+\left(\bar{G}^{r} \sigma_{e \alpha}^{<}-\sigma_{e \alpha}^{<} G^{a}+\bar{G}^{<} \sigma_{e \alpha}^{a}-\sigma_{e \alpha}^{r} G^{<}\right) \delta_{\alpha \beta}\right] .
\end{aligned}
$$

Note that we have simplified the notation and use $G^{r}$ to denote the equilibrium Green's function instead of $G_{0}^{r}$. This applies to other Green's functions and self-energies. Whenever we have two energy variables such as $G^{<}\left(E_{+}, E\right)$ it refers to a nonequilibrium situation. Moreover, in the above equation, $g_{\alpha}^{\gamma}=g_{\alpha}^{\gamma}(\Omega)$ and $\sigma_{e \alpha}^{\gamma}=\sigma_{e \alpha}^{\gamma}(\Omega)$. To make the expression of dynamic conductance simpler, we will use the wideband limit (WBL), where the linewidth function is independent of energy. It is straightforward to extend it to a non-WBL case. Under WBL, Eq. (28) gives

$$
\sigma_{e \alpha}^{r, a}=0
$$

and

$$
\sigma_{e \alpha}^{<}=\frac{i q}{\Omega} \Gamma_{e \alpha}(f-\bar{f}) .
$$

So the first order correction of the Green's function is

$$
g_{\alpha}^{r, a}(\Omega)=\bar{G}^{r, a}\left[u_{\alpha}(\Omega)+\sigma_{e p \alpha}^{r, a}\right] G^{r, a},
$$

where $\sigma_{e p \alpha}^{r, a}(\Omega)=\frac{\partial \sigma_{e p}^{r, a}\left(E_{+}, E\right)}{\partial v_{\alpha}(\Omega)}$. Under WBL, the expression of the dynamic conductance can be simplified as

$$
\begin{aligned}
G_{\alpha \beta}(\Omega)= & -q \int \frac{d E}{2 \pi} \operatorname{Tr}\left[i g_{\beta}^{<} \Gamma_{e \alpha}+\bar{G}^{r} \sigma_{e p \beta}^{r} G^{r} \Sigma_{e \alpha}^{<}\right. \\
& +\bar{G}^{r} u_{\beta} G^{r} \Sigma_{e \alpha}^{<}-\bar{G}^{a} \sigma_{e p \beta}^{a} G^{a} \bar{\Sigma}_{e \alpha}^{<} \\
& \left.-\bar{G}^{a} u_{\beta} G^{a} \bar{\Sigma}_{e \alpha}^{<}+\sigma_{e \alpha}^{<}\left(\bar{G}^{r}-G^{a}\right) \delta_{\alpha \beta}\right] .
\end{aligned}
$$

Using the relation

$$
\begin{aligned}
{\left[\bar{G}^{r}\right]^{-1}-\left[G^{a}\right]^{-1} } & =\Omega+\Sigma^{a}-\bar{\Sigma}^{r} \\
& =\Omega+i \Gamma_{e}+\left(\Sigma_{e p}^{a}-\bar{\Sigma}_{e p}^{r}\right),
\end{aligned}
$$

where $\Gamma_{e}=\sum_{\alpha} \Gamma_{e \alpha}$, we have

$$
\begin{aligned}
G^{a}-\bar{G}^{r} & =\bar{G}^{r}\left[\Omega+i \Gamma_{e}+\left(\Sigma_{e p}^{a}-\bar{\Sigma}_{e p}^{r}\right)\right] G^{a} \\
& =G^{a}\left[\Omega+i \Gamma_{e}+\left(\Sigma_{e p}^{a}-\bar{\Sigma}_{e p}^{r}\right)\right] \bar{G}^{r} .
\end{aligned}
$$

Substituting the above equation into Eq. (52), we find

$$
\begin{aligned}
G_{\alpha \beta}(\Omega)= & -q \int \frac{d E}{2 \pi} \operatorname{Tr}\left[i \bar{G}^{r}\left(\sigma_{e \beta}^{<}+\sigma_{e p \beta}^{<}\right) G^{a} \Gamma_{e \alpha}\right. \\
& +\bar{G}^{r}\left(\sigma_{e p \beta}^{r}+u_{\beta}\right) G^{r} \Sigma_{e \alpha}^{<}-\bar{G}^{a}\left(\sigma_{e p \beta}^{a}+u_{\beta}\right) G^{a} \bar{\Sigma}_{e \alpha}^{<} \\
& +i \bar{G}^{r}\left(\sigma_{e p \beta}^{r}+u_{\beta}\right) G^{<} \Gamma_{e \alpha}+i \bar{G}^{<}\left(\sigma_{e p \beta}^{a}+u_{\beta}\right) G^{a} \Gamma_{e \alpha} \\
& \left.-\bar{G}^{r} \sigma_{e \alpha}^{<} G^{a}\left(\Omega+i \Gamma_{e}+\Sigma_{e p}^{a}-\bar{\Sigma}_{e p}^{r}\right) \delta_{\alpha \beta}\right] .
\end{aligned}
$$

Now we derive the equation which determines the characteristic potential $u_{\alpha}(\Omega)$. The Fourier transform of the Poisson equation under ac bias voltage is given by

$$
\nabla^{2} U(x)=-4 \pi \rho(\Omega)(x)=-4 \pi i q \int \frac{d E}{2 \pi}\left[G^{<}\left(E_{+}, E\right)\right]_{x x} .
$$

With the Poisson equation at equilibrium

$$
\nabla^{2} U_{e q}(x)=-4 \pi \rho_{0}(x)=-4 \pi i q \int \frac{d E}{2 \pi}\left[G^{<}(E)\right]_{x x},
$$

we find the relation between the induced charge distribution $\delta \rho_{\text {ind }}=\rho(\Omega)-\rho_{0}$ and first order correction of Coulomb potential $U_{1}$ due to ac voltage

$\nabla^{2} U_{1}=-4 \pi \delta \rho_{\text {ind }}(\Omega)=-4 \pi i q \int \frac{d E}{2 \pi}\left[g^{<}\left(E_{+}, E\right)\right]_{x x}$.

Taking the derivative with respect to $v_{\alpha}(\Omega)$ on both sides of the above equation, we find (within WBL)

$$
\begin{aligned}
\nabla^{2} u_{\alpha}= & -4 \pi i q \int \frac{d E}{2 \pi}\left[\frac{\partial g^{<}\left(E_{+}, E\right)}{\partial v_{\alpha}}\right]_{x x} \\
= & -4 \pi i q \int \frac{d E}{2 \pi}\left[\bar{G}^{r}\left(u_{\alpha}+\sigma_{e p \alpha}^{r}\right) G^{<}\right. \\
& \left.+\bar{G}^{<}\left(u_{\alpha}+\sigma_{e p \alpha}^{a}\right) G^{a}+\bar{G}^{r}\left(\sigma_{e \alpha}^{<}+\sigma_{e p \alpha}^{<}\right) G^{a}\right]_{x x} .
\end{aligned}
$$

Setting $u_{\alpha}=0$ on the right-hand side of Eq. (59), we obtain the generalized injectivity $d n_{\alpha} / d E$ in the presence of phonon,

$$
\begin{aligned}
d n_{\alpha} / d E= & i \int \frac{d E}{2 \pi}\left[\bar{G}^{r} \sigma_{e p \alpha}^{r}(0) G^{<}+\bar{G}^{<} \sigma_{e p \alpha}^{a}(0) G^{a}\right. \\
& \left.+\bar{G}^{r}\left(\sigma_{e \alpha}^{<}+\sigma_{e p \alpha}^{<}(0)\right) G^{a}\right]_{x x},
\end{aligned}
$$

where $\sigma_{e p \alpha}^{\gamma}(0)$ with $\gamma=r, a,<$ denotes the phonon self-energy in the absence of Coulomb interaction. Here the generalized injectivity $d n_{\alpha} / d E$ describes the density of states inside the scattering region due to the injection of electron in the $\alpha$ lead [11]. Since $g^{<}\left(E_{+}, E\right)$ depends on external bias $v_{\alpha}$ and Coulomb potential $U_{1}$ [see Eqs. (26) and (27)], we have

$$
i g^{<}\left(E_{+}, E\right)=q \sum_{\alpha} d n_{\alpha} / d E v_{\alpha}+q M U_{1}
$$

in the Thomas-Fermi approximation [11], where $d n_{\alpha} / d E=$ $\partial g^{<} / \partial v_{\alpha}$ and $M$ is a Lindhard response function to be determined. From Eq. (58), we see that if we shift $v_{\alpha}$ by a constant amount $v_{0}, U_{1}$ shifts by the same constant and $g^{<}$remains the same. Under this voltage shift, we immediately find from Eq. (61) $M=-\sum_{\alpha} d n_{\alpha} / d E=d n / d E$. So the Poisson-like equation can be cast into the familiar form

$$
\nabla^{2} u_{\alpha}=-4 \pi q^{2} \frac{d n_{\alpha}}{d E}+4 \pi q^{2} \frac{d n}{d E} u_{\alpha} .
$$

The physical meaning of the right-hand side of Eq. (62) is clear: the first term is the injected charge density, while the second term is the induced charge density due to the Coulomb interaction.

Once we have all the Green's functions and self-energies, we can solve for the characteristic potential $u_{\alpha}$ and hence 
the dynamic conductance. In the following, we will show that our formalism satisfies two fundamental requirements for transport in the presence of phonon, i.e., current conservation and gauge invariance. Mathematically, they correspond to $\sum_{\alpha} G_{\alpha \beta}=0$ and $\sum_{\beta} G_{\alpha \beta}=0$.

\section{Current conservation and Gauge invariance}

Before we proceed to show the current conservation and gauge invariance for inelastic ac transport, we want to discuss the basic assumption in the quantum transport of open systems. To deal with quantum transport for an open system such as a two probe system, we always divide the system into scattering region and two semi-infinite leads, where the potential landscape of the lead is assumed to be constant or a periodic function. With this assumption, we can calculate the wave function analytically in the lead region from which the self-energy of the lead in the NEGF approach can be calculated and the scattering matrix in the scattering matrix approach can be constructed. In other words, using this assumption the scattering problem of an open system with infinite degree of freedom can be reduced to a problem of a closed system with finite degree of freedom. In addition, applied ac or dc bias is assumed to shift the potential landscape of the lead by a constant amount (called adiabatic approximation) [15]. The assumption that the potential landscape of the lead is a constant or a periodic function implies that the Coulomb interaction is screened in the lead so that the electric field of the lead is always zero [11]. From Gauss's theorem, the total charge $Q(t)$ inside of the scattering region is always zero, i.e., $Q(t)=0$, although the electrons might be re-distributed in the scattering region due to the existence of the bias voltage. From the continuity equation, we have $\sum_{\alpha} I_{\alpha}+\partial_{t} Q(t)=0$; we conclude that $\sum_{\alpha} I_{\alpha}=0$ as a result of Coulomb interaction. In order to make this assumption valid in practice, we assume that the scattering region is large enough so that the boundaries along the transport directions are deep inside the leads where the electrons are assumed to obey equilibrium distribution $f_{L / R}=$ $\frac{1}{e^{\left(E-\mu_{L / R}-q V_{L / R}\right) / k_{B} T}+1}$. Here $\mu_{L / R}$ are the chemical potentials of left/right leads and $V_{L / R}$ are the bias potentials added at left/right leads.

In the following, we will first derive the continuity equation on the operator level and discuss its implication on current conservation. We then prove explicitly that the current conservation is satisfied in the presence of phonon for both dc and ac cases.

\section{Current conservation on the operator level}

First of all, we give a derivation of the continuity equation on the operator level. Using the Heisenberg equation of motion, one finds

$$
\begin{aligned}
\frac{d \hat{N}_{\alpha}}{d t} & =-i\left[\hat{N}_{\alpha}, \hat{H}\right]=-i\left[\hat{N}_{\alpha}, \hat{H}_{T}\right] \\
& =\sum_{k n}\left(t_{k \alpha n} \hat{C}_{k \alpha}^{\dagger} \hat{d}_{n}-t_{k \alpha n}^{*} \hat{d}_{n}^{\dagger} \hat{C}_{k \alpha}\right),
\end{aligned}
$$

where $\hat{N}_{\alpha}=\sum_{k} \hat{C}_{k \alpha}^{\dagger} \hat{C}_{k \alpha}$ is the number operator for the electron in the lead $\alpha$ that commutes with $\hat{H}_{\text {lead }}, \hat{H}_{e}, \hat{H}_{p}$, and
$\hat{H}_{e p}$. Defining $\hat{N}_{\text {scat }}=\sum_{n} \hat{d}_{n}^{\dagger} \hat{d}_{n}$ the number operator for the electron in the scattering region, we have

$$
\begin{aligned}
\frac{d \hat{N}_{\text {scat }}}{d t} & =-i\left[\hat{N}_{\text {scat }}, \hat{H}\right]=-i\left[\hat{N}_{\text {scat }}, \hat{H}_{T}\right] \\
& =\sum_{k \alpha n}\left(-t_{k \alpha n} \hat{C}_{k \alpha}^{\dagger} \hat{d}_{n}+t_{k \alpha n}^{*} \hat{d}_{n}^{\dagger} \hat{C}_{k \alpha}\right),
\end{aligned}
$$

from which we obtain the continuity equation on the operator level in the presence of phonon

$$
\sum_{\alpha} \frac{d \hat{N}_{\alpha}}{d t}+\frac{d \hat{N}_{\text {scat }}}{d t}=0 .
$$

Taking the quantum average we have the usual continuity equation in the presence of phonon

$$
\sum_{\alpha} I_{\alpha}+\frac{\partial Q}{\partial t}=0,
$$

where $Q$ is the total charge in the scattering region. By including the Coulomb interaction, we solve the following Poisson equation:

$$
\nabla^{2} U(x, t)=-4 \pi \rho(x, t),
$$

with the requirement that the electric field is zero on the boundary. From Gauss's theorem, we have $Q(t)=\int d x \rho(x, t)=0$ or $\sum_{\alpha} I_{\alpha}=0$ from Eq. (66). This shows that the ac current in the presence of phonon is conserved.

\section{Current conservation in dc case}

Now we show explicitly that the current is conserved in the dc case within SCBA. The general expression for current in the dc case is given by

$$
\begin{aligned}
I_{\alpha} & =-q \int \frac{d E}{2 \pi} \operatorname{Tr}\left[G^{r} \Sigma_{e \alpha}^{<}+G^{<} \Sigma_{e \alpha}^{a}+\text { c.c. }\right] \\
& =-q \int \frac{d E}{2 \pi} \operatorname{Tr}\left[\Sigma_{e \alpha}^{<} G^{>}-\Sigma_{e \alpha}^{>} G^{<}\right] .
\end{aligned}
$$

Note that the total self-energy in a two-probe system with electron-phonon interaction can be written as

$$
\Sigma_{\text {tot }}^{\gamma}=\Sigma_{e L}^{\gamma}+\Sigma_{e R}^{\gamma}+\Sigma_{e p}^{\gamma} \quad(\gamma=>,<, r, a) .
$$

The total electron current can be written as

$$
\begin{aligned}
\sum_{\alpha} I_{\alpha}= & -q \int \frac{d E}{2 \pi} \operatorname{Tr}\left[\sum_{\alpha} \Sigma_{e \alpha}^{<} G^{>}-\sum_{\alpha} \Sigma_{e \alpha}^{>} G^{<}\right] \\
= & -q \int \frac{d E}{2 \pi} \operatorname{Tr}\left[\Sigma_{\text {tot }}^{<} G^{>}-\Sigma_{\text {tot }}^{>} G^{<}\right] \\
& -q \int \frac{d E}{2 \pi} \operatorname{Tr}\left[\Sigma_{e p}^{<} G^{>}-\Sigma_{e p}^{>} G^{<}\right] .
\end{aligned}
$$

Using the relationships

$$
\Sigma_{\text {tot }}^{>}-\Sigma_{\text {tot }}^{<}=\Sigma_{\text {tot }}^{r}-\Sigma_{\text {tot }}^{a}, \quad G^{r} \Gamma_{\text {tot }} G^{a}=G^{a} \Gamma_{\text {tot }} G^{r},
$$

it is straightforward to show that the first term of Eq. (70) is zero. Within SCBA, the self-energies due to the 
electron-phonon interaction are $[61,62]$

$$
\begin{aligned}
\Sigma_{e p}^{<}(E)= & \sum_{\nu}\left[n_{\nu} g^{\nu} G^{<}\left(E-\hbar \omega_{\nu}\right) g^{\nu \dagger}\right. \\
& \left.+\left(n_{\nu}+1\right) g^{\nu} G^{<}\left(E+\hbar \omega_{\nu}\right) g^{\nu \dagger}\right], \\
\Sigma_{e p}^{>}(E)= & \sum_{\nu}\left[\left(n_{v}+1\right) g^{\nu} G^{>}\left(E-\hbar \omega_{\nu}\right) g^{\nu \dagger}\right. \\
& \left.+n_{\nu} g^{\nu} G^{>}\left(E+\hbar \omega_{\nu}\right) g^{\nu \dagger}\right] .
\end{aligned}
$$

Plugging these self-energies into Eq. (70), it is easy to show that

$$
\int d E \operatorname{Tr}\left[\Sigma_{e p}^{<} G^{>}-\Sigma_{e p}^{>} G^{<}\right]=0
$$

Hence the dc current is conserved within the SCBA.

In general, the self-energies due to the leads depend on $E-q v_{\alpha}$. By including the Coulomb interaction, the electron Green's functions depend on $E-q U$ as well as self-energies due to the lead and electron-phonon coupling, where $U$ is the Coulomb potential. Thus the self-energies due to electron-phonon coupling depend on $E-q U$ as well since they contain electron Green's function [see Eq. (71)]. If all the external biases are shifted by a constant amount $v_{0}, U$ will also be shifted by $v_{0}$. Hence by changing the variable $E$ to $E+q v_{0}$ in the energy integration in Eq. (68), the current remains unchanged. Therefore, the gauge-invariant condition is automatically satisfied if Coulomb interaction is included.

\section{Current conservation and gauge invariance in ac case}

Now we will prove the continuity equation explicitly in the ac case, i.e., we need to prove

$$
\sum_{\alpha} G_{\alpha \beta}(\Omega)=i q \int \frac{d E}{2 \pi} \operatorname{Tr}\left[g^{<}\left(E_{+}, E\right)\right] .
$$

As we have discussed previously, this equation along with the boundary condition of the Poisson equation will ensure the current conservation. Since the phonon self-energy $\sigma_{e p}^{\gamma}(\gamma=$ $r, a,<)$ cannot be solved explicitly, we will use a perturbative approach by expanding the electron-phonon coupling strength $\left|g^{v}\right|^{2}$ order by order and show that the current is conserved to all orders of $\left|g^{\nu}\right|^{2}$ [for $g^{\nu}$, see Eq. (34)]. For instance, we have

$$
g^{<}=g^{<(0)}+g^{<(1)}+g^{<(2)}+\cdots,
$$

where $g^{<(0)}$ is the lesser Green's function without phonon and $g^{<(n)}$ is the $n$th order correction to the lesser Green's function, i.e., the term containing $\left|g^{\nu}\right|^{2 n}$.

To simplify the proof, we will use the WBL and start with Eq. (55). From the Dyson equation, we can expand the Green's function to the first order of the phonon coupling strength,

$$
G^{r}=G_{e}^{r}+G_{e}^{r} \Sigma_{e p}^{r} G_{e}^{r}
$$

where

$$
G_{e}^{r}=\frac{1}{E-H_{0}-U_{e q}-\Sigma_{e}^{r}}
$$

is the retarded Green's function in the absence of phonon. We first show that the current is conserved to the first order of the electron-phonon coupling strength. So we will keep only the zeroth and the first order terms in $\Sigma_{e p}^{\gamma}$ and $\sigma_{e p}^{\gamma}$ (with $\gamma=r, a,<)$ in Eq. (55). Similar expansion can be done on Eqs. (26) and (27); we find

$$
\begin{aligned}
g_{\alpha}^{r, a}= & \left(\bar{G}_{e}^{r, a}+\bar{G}_{e}^{r, a} \bar{\Sigma}_{e p}^{r, a} \bar{G}_{e}^{r, a}\right)\left(u_{\alpha}+\sigma_{e p}^{r, a}\right) \\
& \times\left(G_{e}^{r, a}+G_{e}^{r, a} \Sigma_{e p}^{r, a} G_{e}^{r, a}\right)
\end{aligned}
$$

and

$$
\begin{aligned}
g_{\alpha}^{<}= & \left(\bar{G}_{e}^{r}+\bar{G}_{e}^{r} \bar{\Sigma}_{e p}^{r} \bar{G}_{e}^{r}\right)\left(\bar{\Sigma}_{e}^{<}+\bar{\Sigma}_{e p}^{<}\right) g_{\alpha}^{a} \\
& +\left(\bar{G}_{e}^{r}+\bar{G}_{e}^{r} \bar{\Sigma}_{e p}^{r} \bar{G}_{e}^{r}\right)\left(\sigma_{e \alpha}^{<}+\sigma_{e p \alpha}^{<}\right)\left(G_{e}^{a}+G^{a} \Sigma_{e p}^{a} G_{e}^{a}\right) \\
& +g_{\alpha}^{r}\left(\Sigma_{e}^{<}+\Sigma_{e p}^{<}\right)\left(G_{e}^{a}+G_{e}^{a} \Sigma_{e p}^{a} G_{e}^{a}\right) .
\end{aligned}
$$

The ac conductance is expanded in terms of electronphonon coupling strength,

$$
G_{\alpha \beta}=G_{\alpha \beta}^{(0)}+G_{\alpha \beta}^{(1)}+G_{\alpha \beta}^{(2)}+\cdots,
$$

where $G_{\alpha \beta}^{(0)}$ is the conductance in the absence of phonon, while $G_{\alpha \beta}^{(1)}$ corresponds to the conductance of the first order correction due to the phonon.

Substituting Eq. (75), Eq. (77), and Eq. (78) into Eq. (55), and using $\sum_{\beta} u_{\beta}(\Omega)=1$, we have

$$
\begin{aligned}
\sum_{\alpha} G_{\alpha \beta}^{(0)}= & -q \int \frac{d E}{2 \pi} \operatorname{Tr}\left[\left(\bar{G}_{e}^{r}-G_{e}^{a}\right) \sigma_{e \beta}^{<}+\bar{G}_{e}^{r} u_{\beta} G_{e}^{r} \Sigma_{e}^{<}\right. \\
& -\bar{\Sigma}_{e}^{<} \bar{G}_{e}^{a} u_{\beta} G_{e}^{a}+i \bar{G}_{e}^{r}\left(u_{\beta} G_{e}^{r} \Sigma_{e}^{<}+\sigma_{e \beta}^{<}\right. \\
& \left.\left.+\bar{\Sigma}_{e}^{<} \bar{G}_{e}^{a} u_{\beta}\right) G_{e}^{a} \Gamma_{e}\right]
\end{aligned}
$$

and

$$
\sum_{\alpha} G_{\alpha \beta}^{(1)}=-q \int \frac{d E}{2 \pi} \operatorname{Tr}\left[A_{1}+A_{2}+A_{3}+A_{4}+A_{5}\right] .
$$

Here,

$$
\begin{gathered}
A_{1}=\left(\bar{G}_{e}^{r} \bar{\Sigma}_{e p}^{r} \bar{G}_{e}^{r}-G_{e}^{a} \Sigma_{e p}^{a} G_{e}^{a}\right) \sigma_{e \beta}^{<}, \\
A_{2}=\bar{G}_{e}^{r}\left(\sigma_{e p \beta}^{r}+u_{\beta} G_{e}^{r} \Sigma_{e p}^{r}+\bar{\Sigma}_{e p}^{r} \bar{G}_{e}^{r} u_{\beta}\right) G_{e}^{r} \Sigma_{e}^{<} \\
-\bar{\Sigma}_{e}^{<} \bar{G}_{e}^{a}\left(\sigma_{e p \beta}^{a}+u_{\beta} G_{e}^{a} \Sigma_{e p}^{a}+\bar{\Sigma}_{e p}^{a} \bar{G}_{e}^{a} u_{\beta}\right) G_{e}^{a}, \\
A_{3}=i \bar{G}_{e}^{r}\left(\bar{\Sigma}_{e p}^{r} \bar{G}_{e}^{r} u_{\beta} G_{e}^{r} \Sigma_{e}^{<}+u_{\beta} G_{e}^{r} \Sigma_{e p}^{r} G_{e}^{r} \Sigma_{e}^{<}\right. \\
\left.+\sigma_{e p \beta}^{r} G_{e}^{r} \Sigma_{e}^{<}+u_{\beta} G_{e}^{r} \Sigma_{e p}^{<}+u_{\beta} G_{e}^{r} \Sigma_{e}^{<} G_{e}^{a} \Sigma_{e p}^{a}\right) G_{e}^{a} \Gamma_{e},
\end{gathered}
$$

$$
\begin{aligned}
A_{4}= & i \bar{G}_{e}^{r}\left(\bar{\Sigma}_{e p}^{r} \bar{G}_{e}^{r} \bar{\Sigma}_{e}^{<} \bar{G}_{e}^{a} u_{\beta}+\bar{\Sigma}_{e}^{<} \bar{G}_{e}^{a} \bar{\Sigma}_{e p}^{a} \bar{G}_{e}^{a} u_{\beta}\right. \\
& \left.+\bar{\Sigma}_{e p}^{<} \bar{G}_{e}^{a} u_{\beta}+\bar{\Sigma}_{e}^{<} \bar{G}_{e}^{a} \sigma_{e p \beta}^{a}+\bar{\Sigma}_{e}^{<} \bar{G}_{e}^{a} u_{\beta} G_{e}^{a} \Sigma_{e p}^{a}\right) G_{e}^{a} \Gamma_{e},
\end{aligned}
$$

and

$$
A_{5}=i \bar{G}_{e}^{r}\left(\bar{\Sigma}_{e p}^{r} \bar{G}_{e}^{r} \sigma_{e \beta}^{<}+\sigma_{e p \beta}^{<}+\sigma_{e \beta}^{<} G_{e}^{a} \Sigma_{e p}^{a}\right) G_{e}^{a} \Gamma_{e} .
$$

Using the relationship

$$
\begin{aligned}
i G_{e}^{a} \Gamma_{e} \bar{G}_{e}^{r} & =G_{e}^{a}-\bar{G}_{e}^{r}-\Omega G_{e}^{a} \bar{G}_{e}^{r}, \\
G_{e}^{a}-G_{e}^{r} & =i G_{e}^{r} \Gamma_{e} G_{e}^{a}=i G_{e}^{a} \Gamma_{e} G_{e}^{r},
\end{aligned}
$$


it is straightforward but tedious to show that

$$
\sum_{\alpha} G_{\alpha \beta}^{(0)}=q \Omega \int \frac{d E}{2 \pi} \operatorname{Tr}\left[g_{\beta}^{<(0)}\right]=0
$$

and

$$
\sum_{\alpha} G_{\alpha \beta}^{(1)}=q \Omega \int \frac{d E}{2 \pi} \operatorname{Tr}\left[g_{\beta}^{<(1)}\right]=0 .
$$

One can easily push it to higher order and show that

$$
\sum_{\alpha} G_{\alpha \beta}^{(n)}=q \Omega \int \frac{d E}{2 \pi} \operatorname{Tr}\left[g_{\beta}^{<(n)}\right]=0
$$

for $n>1$. Finally, we obtain that

$$
\sum_{\alpha} G_{\alpha \beta}=0,
$$

which is the expected result.

Now we show that the gauge-invariant condition is satisfied. From Eq. (48), we have

$$
\begin{aligned}
\sum_{\beta} G_{\alpha \beta}^{(0)}= & -q \int \frac{d E}{2 \pi} \operatorname{Tr}\left[\left(\bar{G}_{e}^{r}-G_{e}^{a}\right) \sigma_{e \alpha}^{<}+i\left(f \bar{G}_{e}^{r} G_{e}^{r}\right.\right. \\
& \left.\left.-\bar{f} \bar{G}_{e}^{a} G_{e}^{a}+\bar{G}_{e}^{r} G_{e}^{<}+\bar{G}_{e}^{<} G_{e}^{a}+\bar{G}_{e}^{r} \sigma_{e}^{<} G_{e}^{a}\right) \Gamma_{e \alpha}\right] .
\end{aligned}
$$

Here,

$$
\sigma_{e}^{<}=\sigma_{e}^{<}(\Omega)=\sum_{\beta} \sigma_{e \beta}^{<}(\Omega) .
$$

Similar to the current conservation, we have the expression of first order correction to the conductance in the presence of phonon

$$
\sum_{\beta} G_{\alpha \beta}^{(1)}=-q \int \frac{d E}{2 \pi} \operatorname{Tr}\left[B_{1}+B_{2}+B_{3}+B_{4}+B_{5}\right],
$$

where

$$
\begin{gathered}
B_{1}=\left(\bar{G}_{e}^{r} \bar{\Sigma}_{e p}^{r} \bar{G}_{e}^{r}-G_{e}^{a} \Sigma_{e p}^{a} G_{e}^{a}\right) \sigma_{e \alpha}^{<}, \\
B_{2}=\bar{G}_{e}^{r}\left(\sigma_{e p}^{r}+G_{e}^{r} \Sigma_{e p}^{r}+\bar{\Sigma}_{e p}^{r} \bar{G}_{e}^{r}\right) G_{e}^{r} \Sigma_{e \alpha}^{<} \\
-\bar{G}_{e}^{a}\left(\sigma_{e p}^{a}+G_{e}^{a} \Sigma_{e p}^{a}+\bar{\Sigma}_{e p}^{a} \bar{G}_{e}^{a}\right) G_{e}^{a} \bar{\Sigma}_{e \alpha}^{<}, \\
B_{3}=i \bar{G}_{e}^{r}\left(\sigma_{e p}^{r} G_{e}^{r} \Sigma_{e}^{<}+G_{e}^{r} \Sigma_{e}^{<} G_{e}^{a} \Sigma_{e p}^{a}+G_{e}^{r} \Sigma_{e p}^{<}\right. \\
\left.+G_{e}^{r} \Sigma_{e p}^{r} G_{e}^{r} \Sigma_{e}^{<}+\bar{\Sigma}_{e p}^{r} \bar{G}_{e}^{r} G_{e}^{r} \Sigma_{e}^{<}\right) G_{e}^{a} \Gamma_{e \alpha}, \\
B_{4}=i \bar{G}_{e}^{r}\left(\bar{\Sigma}_{e}^{<} \bar{G}_{e}^{a} \sigma_{e p}^{a}+\bar{\Sigma}_{e}^{<} \bar{G}_{e}^{a} \bar{\Sigma}_{e p}^{a} \bar{G}_{e}^{a}+\bar{\Sigma}_{e p}^{<} \bar{G}_{e}^{a}\right. \\
\left.+\bar{\Sigma}_{e}^{<} \bar{G}_{e}^{a} G_{e}^{a} \Sigma_{e p}^{a}+\bar{\Sigma}_{e p}^{r} \bar{G}_{e}^{r} \bar{\Sigma}_{e}^{<} \bar{G}_{e}^{a}\right) G_{e}^{a} \Gamma_{e \alpha}, \\
B_{5}=i \bar{G}_{e}^{r}\left(\sigma_{e p}^{<}+\sigma_{e}^{<} G_{e}^{a} \Sigma_{e p}^{a}+\bar{\Sigma}_{e p}^{r} \bar{G}_{e}^{r} \sigma_{e}^{<}\right) G_{e}^{a} \Gamma_{e \alpha} .
\end{gathered}
$$

Using the relationship of Eq. (87) and $\sum_{\beta} u_{\beta}(\Omega)=1$, it is straightforward to show

$$
\sum_{\beta} G_{\alpha \beta}^{(0)}=0
$$

and

$$
\sum_{\beta} G_{\alpha \beta}^{(1)}=0
$$

So far, we have proved that the zeroth order and first order of $\sum_{\beta} G_{\alpha \beta}$ are zero. In the same way, we can prove that all the higher order terms are zero although it is tedious but straightforward. Finally, we have

$$
\sum_{\beta} G_{\alpha \beta}=0 .
$$

That is the condition of gauge invariance.

\section{E. First principles calculation}

We note that a first principles formalism of dc quantum transport by doing density function theory (DFT) calculation within nonequilibrium Green's function theory (NEGF-DFT) has been well established and extended to include the electronphonon interaction [40,52]. First principles investigations have also been carried out using the NEGF-DFT approach for molecular devices in the presence of ac bias [65]. In view of the above progress, our formalism presented in this paper can in principle be implemented within NEGF-DFT framework so that inelastic ac transport calculation can be carried out from first principles. To do this, we start from Eq. (5) where the potentials due to the exchange and correlation are included that are functional of charge density which is given before the iteration. From Eqs. (5), (36), and (37) we find the equilibrium Green's function from which we can construct the new charge density. This in turn gives the new potential due to exchange and correlation. We then solve the Poisson-like Eq. (62) to find the characteristic potential which gives a new Hamiltonian. We repeat this iteration until it reaches the self-consistency. Finally, we use Eq. (55) to calculate the dynamic conductance in the presence of phonon.

So far, we have treated the electron-phonon coupling strength $g^{v}$ as a constant. Actually both electron-phonon coupling and phonon spectrum depend on the external bias in the dc case and the driving frequency in the ac linear regime. In the presence of phonon, we assume that the phonon exists only in the scattering region. To calculate the phonon spectrum, one has to diagonalize the Hessian matrix (dynamic matrix) which is constructed from the second derivative of the total energy of the scattering region with respect to the position of the atom (for details, see Chaps. 4 and 5 in Ref. [66]). Importantly, the total energy is a functional of charge density which depends on external bias in the dc case and the driving frequency in the ac case. As a result, the phonon frequency and phonon eigenvector depend on the external bias in the dc case. The electron-phonon coupling is also related to the phonon frequency and corresponding eigenvector. It was found in Refs. [52,56] that due to the external bias many phonon frequencies are renormalized between $10 \%$ and $30 \%$ for molecular junctions, while the electron-phonon coupling constant is affected significantly by an order of magnitude. Since the Hessian matrix depends on the driving frequency of the external bias in the ac case, the phonon frequency as well as electron-phonon coupling may also be sensitive to the 
driving frequency. To address this issue quantitatively, a first principles calculation has to be performed. In this paper we have laid down the foundation of ac transport theory in the presence of phonon; the implementation of this formalism in first principles calculation will be the subject of future work.

Now we wish to make some comments on the DFT used in NEGF-DFT. In the dc case, the leads are in equilibrium with well defined Fermi distribution functions. However, the scattering region is out of equilibrium with the charge density expressed in terms of nonequilibrium lesser Green's function that depends on external bias. Therefore, in the formalism of NEGF-DFT, the density matrix or charge density is constructed at nonequilibrium using nonequilibrium Green's functions. Due to this nonequilibrium nature, there is no minimization principle to converge the charge density in open systems [67]. The above discussion applies to the ac case as well except that one has to use time-dependent DFT (TDDFT) [68] instead of static DFT. To reduce the computational complexity while still capturing the essential physics, people usually use the adiabatic local density approximation for the exchange and correlation functionals in TDDFT. This scheme has been used to predict transient dynamics of molecular junctions [34]. Recently, the applicability of DFT in the open systems has been put on a more rigorous basis [69].

\section{F. Inelastic electron admittance spectroscopy}

In the dc situation, inelastic electron tunneling spectroscopy (IETS), which is the second derivative of the current with respect to the bias voltage, is widely used as a powerful tool to identify the molecular vibrational modes and the electron-phonon coupling strength. Similarly, in the ac situation we will show below that the inelastic electron admittance spectroscopy (IEAS), which is the second derivative of the admittance with respect to ac frequency, can have a similar functionality but with the frequency as an extra handle.

To demonstrate the feasibility of IEAS, we focus on the conductance up to the first order in electron-phonon coupling strength, which gives

$$
\begin{aligned}
G_{L R}= & -q^{2} \int \frac{d E}{2 \pi} \frac{f-\bar{f}}{\Omega}\left\{\operatorname{Tr}\left[B_{1}\right]+\sum_{\nu} \operatorname{Tr}\left[B_{2}\right]\right\} \\
& -q^{2} \int \frac{d E}{2 \pi} \frac{f-\bar{f}}{\Omega} \sum_{\nu} f\left(E+\omega_{\nu}\right) \operatorname{Tr}\left[B_{3}\right],
\end{aligned}
$$

where $f=f(E), \bar{f}=f(E+\Omega)$, and $B_{i}$ are expressed in terms of the Green's function

$$
\begin{gathered}
B_{1}=i \Omega \bar{G}_{e}^{r} u_{R} G_{e}^{a} \Gamma_{e L}-\bar{G}_{0 e}^{r} \Gamma_{e R} G_{e}^{a} \Gamma_{e L} \\
+i \Omega \bar{G}_{e}^{r} u_{R} G_{e}^{a}\left(\Sigma_{e p}^{a} G_{e}^{a} \Gamma_{e L}+\Gamma_{e L} \bar{G}_{e}^{r} \bar{\Sigma}_{e p}^{r}\right) \\
-\bar{G}_{e}^{r} \Gamma_{e R} G_{e}^{a}\left(\Sigma_{e p}^{a} G_{e}^{a} \Gamma_{e L}+\Gamma_{e L} \bar{G}_{e}^{r} \bar{\Sigma}_{e p}^{r}\right), \\
B_{2 v}=i \Omega G_{e}^{a} \Gamma_{e L} \bar{G}_{e}^{r} g^{\nu}\left(\bar{G}_{e-}^{r} u_{R} G_{e-}^{r}+\bar{G}_{e-}^{a} u_{R} G_{e-}^{a}\right) g^{\nu \dagger} / 2,
\end{gathered}
$$

and

$$
\begin{aligned}
B_{3 v}= & {\left[i \Omega G_{e}^{a} \Gamma_{e L} \bar{G}_{e}^{r} g^{\nu} \bar{G}_{e+}^{r} u_{R}\left(G_{e+}^{a}-G_{o e+}^{r}\right) g^{\nu \dagger}\right.} \\
& \left.-G_{e}^{a} \Gamma_{e L} \bar{G}_{e}^{r} g^{\nu} \bar{G}_{e+}^{r} \Gamma_{e R} G_{e+}^{a} g^{\nu \dagger}\right] / 2 \\
& +\left[G_{e+}^{a} \Gamma_{e L} \bar{G}_{e+}^{r} g^{\nu} \bar{G}_{e}^{r}\left(i \Omega u_{R}-\Gamma_{R}\right) G_{e}^{a} g^{\nu \dagger}\right] / 2 .
\end{aligned}
$$

In the above equations, $G_{e \pm}^{\gamma}=G_{e}^{\gamma}\left(E \pm \omega_{v}\right)$. From Eqs. (42)(44) we see that the phonon self-energies in general contain Fermi distribution functions through $g^{<}$and $g^{>}$. As a result, we see from Eqs. (55) and (103) that we will have terms involving two Fermi distribution functions $f(E)$ and $f\left(E+\omega_{\nu}\right)$. As we will see below, these terms are very important for inelastic electron admittance spectroscopy.

At zero temperature, Eq. (103) becomes

$$
\begin{aligned}
G_{L R}= & -\frac{q^{2}}{\Omega} \int_{-\Omega}^{0} \frac{d E}{2 \pi}\left\{\operatorname{Tr}\left[B_{1}\right]+\sum_{\nu} \operatorname{Tr}\left[B_{2 v}\right]\right\} \\
& -\frac{q^{2}}{\Omega} \int_{-\Omega}^{0} \frac{d E}{2 \pi} \sum_{\nu} f\left(E+\omega_{\nu}\right) \operatorname{Tr}\left[B_{3 v}\right],
\end{aligned}
$$

where at zero temperature $f\left(E+\omega_{\nu}\right)$ is a step function and we have set the Fermi energy $E_{F}=0$. So the second term in Eq. (107) is zero for $E>-\omega_{\nu}$. On the other hand, the lower and upper limits of the integral require $-\Omega<E<0$. Consequently, the second term does not contribute to the integral when $\Omega<\omega_{\nu}$. As a result, as we vary $\Omega$ the integrand of the second term in Eq. (107) will jump by $\operatorname{Tr}\left[B_{3 v}\right]$ whenever $\Omega$ sweeps through $\omega_{\nu}$. After the integration over energy, the contribution of this term near $\omega_{\nu}$ is proportional to $\left(\Omega-\omega_{v}\right) \operatorname{Tr}\left[B_{3 v}\right]$ giving rise to a discontinuity of $\partial_{\Omega} G_{L R}$ near $\omega_{\nu}$ [see Fig. 3(b)]. Furthermore, from Eq. (106), $\operatorname{Tr}\left[B_{3 v}\right]$ is proportional to the electron-phonon coupling strength. In contrast, the first term in Eq. (107) is a continuous function of $\Omega$ and the numerical calculation in Sec. III also shows that this term changes slowly with frequency $\Omega$. Hence the second derivative of the second term in $G_{L R}$ with respect to $\Omega$ will give peaks at $\Omega=\omega_{\nu}$, while no peaks are contributed from the first term in $G_{L R}$. Thus we have

$$
\frac{\partial^{2} G_{L R}}{\partial \Omega^{2}} \sim \frac{q^{2}}{\Omega} \sum_{\nu} \delta\left(\omega_{v}-\Omega\right) \operatorname{Tr}\left[B_{3 v}(-\Omega)\right] .
$$

So this quantity can be used to analyze the inelastic ac quantum transport which we term as ac inelastic electron admittance spectroscopy (IEAS).

\section{NUMERICAL CALCULATION OF A QUANTUM DOT}

To study dynamic conductance numerically, we consider a single level quantum dot system connected by two leads. All the quantities we use in the following calculation are in the Hartree atomic unit. As we did in formulating the theory, we assume that the electron-phonon interaction exists only in the quantum dot. Furthermore, we assume that there are four vibrational modes in the quantum dot and the vibrational frequencies $\omega_{\nu}$ are $0.003,0.005,0.007$, and 0.009 , and the corresponding electron-phonon coupling strengths $g^{v}$ are 0.09 , $0.07,0.05$, and 0.03 , respectively, and we set the electron self-energy $\Gamma_{e L}=0.2$ and $\Gamma_{e R}=0.3$. 


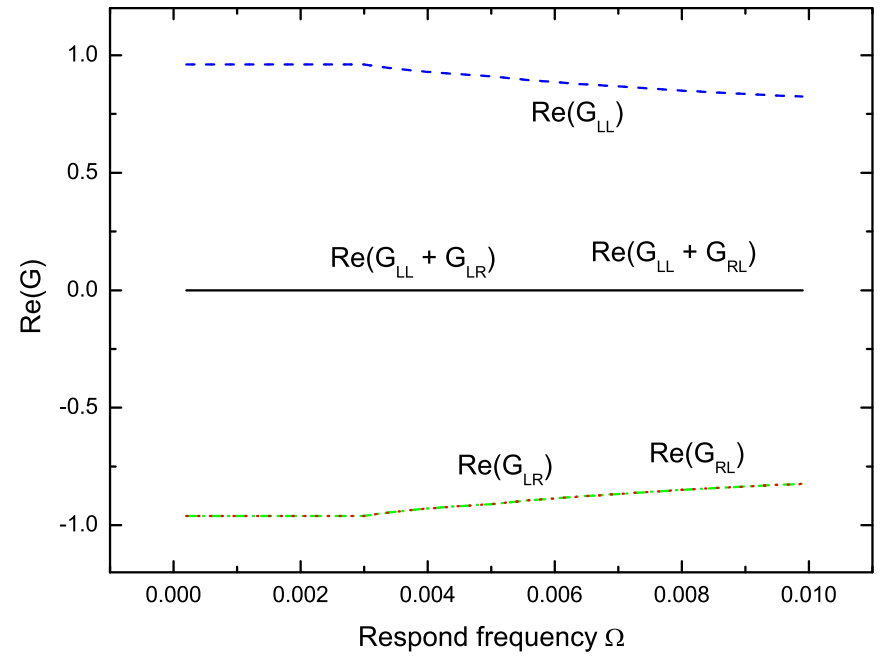

FIG. 1. (Color online) Real part of admittance vs frequency. The blue dash line is for $\operatorname{Re}\left[G_{L L}(\Omega)\right]$, the green dash dot line for $\operatorname{Re}\left[G_{L R}(\Omega)\right]$ and the red dot line for $\operatorname{Re}\left[G_{R L}(\Omega)\right]$ are overlap, and the black solid line is for the real part of $\operatorname{Re}\left(G_{L L}+G_{L R}\right)$ or $\operatorname{Re}\left(G_{L L}+G_{R L}\right)$.

Figures 1 and 2 depict the real and imaginary part of dynamic admittance versus the frequency, respectively. The blue dash line is for $G_{L L}$, the green dash dot line is for $G_{L R}$, and the red dot line is for $G_{R L}$. When the ac frequency exceeds certain phonon frequency, the effect of this phonon mode is activated. So the real part and imaginary part of admittance show piece-wise behaviors. Furthermore, our results show that $G_{L R}$ and $G_{R L}$ are the same as expected. The black solid line is for $G_{L L}+G_{L R}$ or $G_{L L}+G_{R L}$ which confirm the conservations of inelastic ac current, i.e., $G_{L L}+G_{R L}=0$ and $G_{L L}+G_{L R}=0$.

In Fig. 3 we plot the imaginary part of dynamic admittance $\operatorname{Im}\left(G_{L L}\right)$, its first derivative $\frac{\partial \operatorname{Im}\left(G_{L L}\right)}{\partial \Omega}$ and the second derivative $\frac{\partial^{2} \operatorname{Im}\left(G_{L L}\right)}{\partial \Omega^{2}}$ versus the frequency in panels (a), (b), and (c),

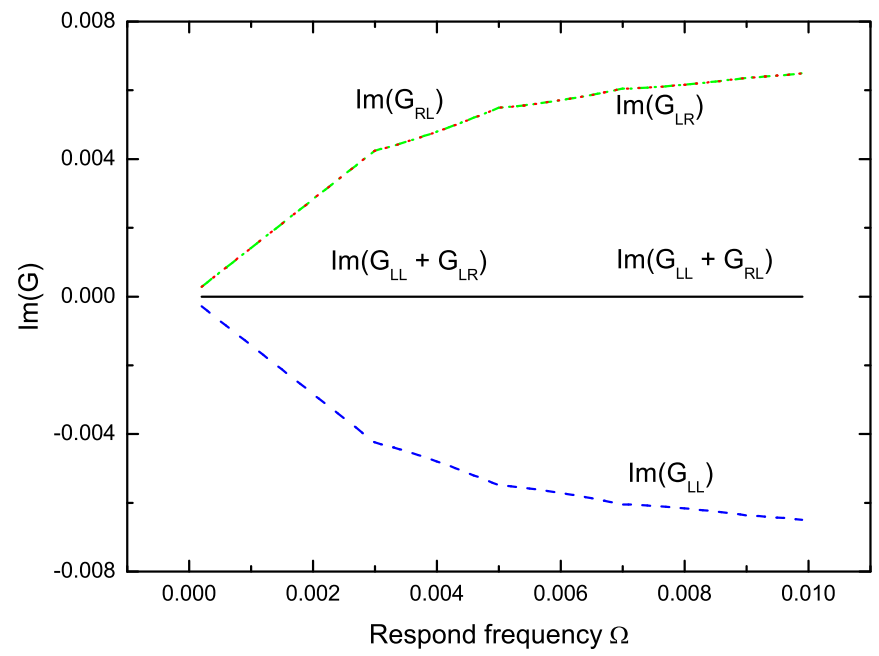

FIG. 2. (Color online) Imaginary part of admittance vs frequency. The blue dash line is for $\operatorname{Im}\left[G_{L L}(\Omega)\right]$, the green dash dot line for $\operatorname{Im}\left[G_{L R}(\Omega)\right]$ and the red dot line for $\operatorname{Im}\left[G_{R L}(\Omega)\right]$ are overlap, and the black solid line is for the real part of $\operatorname{Im}\left(G_{L L}+G_{L R}\right)$ or $\operatorname{Im}\left(G_{L L}+G_{R L}\right)$.

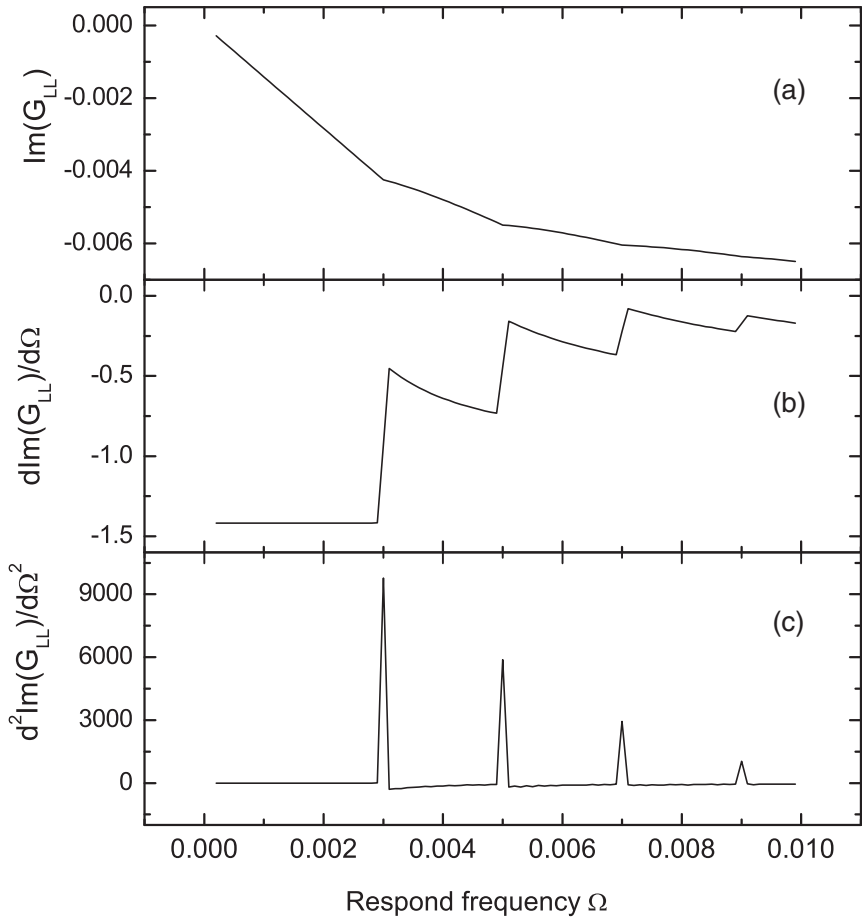

FIG. 3. (a) Imaginary part of admittance, (b) the first derivative of imaginary part of admittance with respective to frequency, and (c) the second derivative of imaginary part of admittance with respective to frequency versus the frequency.

respectively. One can see that $\frac{\partial^{2} \operatorname{Im}\left(G_{L L}\right)}{\partial \Omega^{2}}$ gives a peak at each phonon frequency with the peak height determined by the electron-phonon strength. The larger the strength, the higher the peak. Similar behavior is found for the real part of dynamic admittance $\operatorname{Re}\left(G_{L L}\right)$. This shows that we can use $\frac{\partial^{2} G_{L L}}{\partial \Omega^{2}}$ to acquire the information of electron-phonon interaction including its frequency and coupling strength, making the IEAS a useful tool in studying the inelastic ac transport.

\section{SUMMARY}

In this paper, we developed a theoretical formalism for ac transport with electron-phonon interaction based on the nonequilibrium Green's function method. The Coulomb interaction is included self-consistently so that the currentconserving and gauge-invariant conditions are satisfied. Our formalism can be used for first principles transport calculation within NEGF-DFT formalism. We also proposed that the inelastic electron admittance spectroscopy can be used to probe the influence of the electron-phonon interaction on the dynamic conductance in molecular devices.

\section{ACKNOWLEDGMENTS}

We gratefully acknowledge the support by National Natural Science Foundation of China with Grant No. 11074171 (Y.D.W.) and No. 11374246 (J.W.) and GRF Grant No. HKU 705212P, the UGC grant (Contract No. AoE/P-04/08) from the Government of HKSAR. 
[1] M. A. Ratner, Mater. Today 5, 20 (2002).

[2] J. R. Heath and M. A. Ratner, Phys. Today 56, 43 (2003).

[3] N. A. Zimbovskaya and M. R. Pederson, Phys. Rep. 509, 1 (2011).

[4] T. P. Smith, III, B. B. Goldberg, P. J. Stiles, and M. Heiblum, Phys. Rev. B 32, 2696(R) (1985); T. P. Smith, III, W. I. Wang, and P. J. Stiles, ibid. 34, 2995(R) (1986).

[5] R. A. Webb, S. Washburn, and C. P. Umbach, Phys. Rev. B 37, 8455 (1988).

[6] J. B. Pieper and J. C. Price, Phys. Rev. Lett. 72, 3586 (1994).

[7] W. Chen, T. P. Smith, III, M. Büttiker, and M. Shayegan, Phys. Rev. Lett. 73, 146 (1994).

[8] L. P. Kouwenhoven, A. T. Johnson, N. C. van der Vaart, C. J. P. M. Harmans, and C. T. Foxon, Phys. Rev. Lett. 67, 1626 (1991).

[9] L. P. Kouwenhoven, S. Jauhar, J. Orenstein, P. L. McEuen, Y. Nagamune, J. Motohisa, and H. Sakaki, Phys. Rev. Lett. 73, 3443 (1994).

[10] M. Büttiker, A. Prêtre, and H. Thomas, Phys. Rev. Lett. 70, 4114 (1993).

[11] M. Büttiker, J. Phys.: Condens. Matter 5, 9361 (1993).

[12] T. Gramespacher and M. Buttiker, Phys. Rev. Lett. 81, 2763 (1998).

[13] Y. D. Wei, B. G. Wang, J. Wang, and H. Guo, Phys. Rev. B 60, 16900 (1999).

[14] A. P. Jauho, J. Phys.: Conf. Ser. 35, 313 (2006).

[15] N. S. Wingreen, A.-P. Jauho, and Y. Meir, Phys. Rev. B 48, 8487 (1993).

[16] B. G. Wang, X. A. Zhao, J. Wang, and H. Guo, Appl. Phys. Lett. 74, 2887 (1999).

[17] N. S. Wingreen, K. W. Jacobsen, and J. W. Wilkins, Phys. Rev. B 40, 11834 (1989).

[18] R. H. Blick, R. J. Haug, D. W. van der Weide, K. von Klitzing, and K. Eberl, Appl. Phys. Lett. 67, 3924 (1995).

[19] T. H. Oosterkamp, L. P. Kouwenhoven, A. E. A. Koolen, N. C. van der Vaart, and C. J. P. M. Harmans, Phys. Rev. Lett. 78, 1536 (1997).

[20] A. Fujiwara, Y. Takahashi, and K. Murase, Phys. Rev. Lett. 78, 1532 (1997).

[21] M. P. Anantram and S. Datta, Phys. Rev. B 51, 7632 (1995).

[22] R. J. Schoelkopf, A. A. Kozhevnikov, D. E. Prober, and M. J. Rooks, Phys. Rev. Lett. 80, 2437 (1998).

[23] B. G. Wang, J. Wang, and H. Guo, Phys. Rev. Lett. 82, 398 (1999).

[24] J. Wang, J. Comput. Electron. 12, 343 (2013).

[25] Y. D. Wei and J. Wang, Phys. Rev. B 79, 195315 (2009).

[26] Y. X. Xing, B. Wang, and J. Wang, Phys. Rev. B 82, 205112 (2010).

[27] M. Tahir and A. MacKinnon, Phys. Rev. B 81, 195444 (2010).

[28] D. Kienle, M. Vaidyanathan, and F. Leonard, Phys. Rev. B 81, 115455 (2010).

[29] J. N. Zhuang, L. Zhang, and J. Wang, AIP Adv. 1, 042180 (2011).

[30] L. Zhang, B. Wang, and J. Wang, Phys. Rev. B 86, 165431 (2012).

[31] O. Shevtsov and X. Waintal, Phys. Rev. B 87, 085304 (2013).

[32] Y. J. Yu, B. Wang, and Y. D. Wei, J. Chem. Phys. 127, 169901 (2007).

[33] A. P. Jauho, N. S. Wingreen, and Y. Meir, Phys. Rev. B 50, 5528 (1994).
[34] B. Wang, Y. X. Xing, L. Zhang, and J. Wang, Phys. Rev. B 81, 121103(R) (2010); L. Zhang, Y. X. Xing, and J. Wang, ibid. 86, 155438 (2012); L. Zhang, J. Chen, and J. Wang, ibid. 87, 205401 (2013).

[35] C. Bruder and H. Schoeller, Phys. Rev. Lett. 72, 1076 (1994).

[36] Q. F. Sun, J. Wang, and T. H. Lin, Phys. Rev. B 61, 12643 (2000)

[37] J. C. Cuevas, A. Martin-Rodero, and A. Levy Yeyati, Phys. Rev. B 54, 7366 (1996).

[38] T. C. Au Yeung, W. Z. Shangguan, Q. H. Chen, Y. B. Yu, C. H Kam, and M. C. Wong, Phys. Rev. B 65, 035306 (2001).

[39] R. Lake and S. Datta, Phys. Rev. B 45, 6670 (1992).

[40] J. Taylor, H. Guo, and J. Wang, Phys. Rev. B 63, 121104 (2001); 63, 245407 (2001).

[41] N. Agraï, C. Untiedt, G. Rubio-Bollinger, and S. Vieira, Phys Rev. Lett. 88, 216803 (2002); N. Agrait, C. Untiedt, G. RubioBollinger, and S. Vieira, Chem. Phys. 281, 231 (2002).

[42] A. Arbouet, C. Voisin, D. Christofilos, P. Langot, N. Del Fatti, F. Vallée, J. Lermé, G. Celep, E. Cottancin, M. Gaudry, M. Pellarin, M. Broyer, M. Maillard, M. P. Pileni, and M. Treguer, Phys. Rev. Lett. 90, 177401 (2003).

[43] J. Y. Park, S. Rosenblatt, Y. Yaish, V. Sazonova, H. Ustunel, S. Braig, T. A. Arias, P. W. Brouwer, and P. L. McEuen, Nano Lett. 4, 517 (2004).

[44] Y. C. Chen, M. Zwolak, and M. D. Ventra, Nano Lett. 3, 1691 (2003).

[45] J. Gaudioso, L. J. Lauhon, and W. Ho, Phys. Rev. Lett. 85, 1918 (2000).

[46] S. Sapmaz, P. Jarillo-Herrero, Ya. M. Blanter, C. Dekker, and H. S. J. van der Zant, Phys. Rev. Lett. 96, 026801 (2006).

[47] P. Kral, F. W. Sheard, and F. F. Ouali, Phys. Rev. B 57, 15428 (1998).

[48] J. K. Viljas, J. C. Cuevas, F. Pauly, and M. Hafner, Phys. Rev. B 72, 245415 (2005).

[49] J. T. Lu and J. S. Wang, Phys. Rev. B 76, 165418 (2007).

[50] J. Ren, J. X. Zhu, J. E. Gubernatis, C. Wang, and B. W. Li, Phys. Rev. B 85, 155443 (2012).

[51] K. Haule and J. Bonča, Phys. Rev. B 59, 13087 (1999).

[52] N. Sergueev, D. Roubtsov, and H. Guo, Phys. Rev. Lett. 95, 146803 (2005).

[53] T. Frederiksen, M. Paulsson, M. Brandbyge, and A. P. Jauho, Phys. Rev. B 75, 205413 (2007).

[54] H. Nakamura, K. Yamashita, A. R. Rocha, and S. Sanvito, Phys. Rev. B 78, 235420 (2008)

[55] M. Galperin, M. A. Ratner, and A. Nitzan, Nano Lett. 4, 1605 (2004).

[56] N. Sergueev, A. A. Demkov, and H. Guo, Phys. Rev. B 75 , 233418 (2007).

[57] We note that Refs. [21,38,39] investigated ac transport in the presence of electron-phonon interaction but the self-consistent Coulomb interaction was not considered, which is the key for first principles transport calculation.

[58] L. P. Kadanoff and G. Baym, Quantum Statistical Mechanics (Benjamin, London, 1962), p. 21.

[59] The potentials for exchange and correlation interaction can be easily included for first principles calculation.

[60] D. A. Ryndyk and J. Keller, Phys. Rev. B 71, 073305 (2005).

[61] E. J. McEniry, T. Frederiksen, T. N. Todorov, D. Dundas, and A. P. Horsfield, Phys. Rev. B 78, 035446 (2008).

[62] W. Lee, N. Jean, and S. Sanvito, Phys. Rev. B 79, 085120 (2009). 
[63] T. Ji, Ph.D. thesis, McGill University, 2010.

[64] H. Haug and A. P. Jauho, Quantum Kinetics in Transport and Optics of Semiconductors (Springer, Berlin, 1998).

[65] For a recent review, see Ref. [24].

[66] N. Sergueev, Ph.D. thesis, McGill University, 2005.
[67] Z. Q. Yang, A. Tackett, and M. Di Ventra, Phys. Rev. B 66, 041405 (2002).

[68] E. Runge and E. K. U. Gross, Phys. Rev. Lett. 52, 997 (1984).

[69] X. Zheng, F. Wang, C. Y. Yam, Y. Mo, and G. H. Chen, Phys. Rev. B 75, 195127 (2007). 